\title{
Overview literature on matrix assisted laser desorption ionization mass spectroscopy (MALDI MS): basics and its applications in characterizing polymeric materials
}

\author{
R N JAGTAP* and A H AMBRE \\ Polymer Engineering and Technology Department, University of Mumbai, Institute of Chemical Technology,
} Mumbai 400 019, India

MS received 30 December 2004; revised 11 April 2005

\begin{abstract}
Matrix assisted laser desorption ionization mass spectroscopy (MALDI MS) is a technique which allows the measurement of molecular mass $>\mathbf{2 0 0 , 0 0 0}$ Daltons by ionization and vapourization without degradation. This technique is useful for the mass analysis of synthetic polymers, which have very low volatility. The basic principles of and its applications for polymer characterization have been discussed in this paper. In addition, the possibilities of combining MALDI MS with chromatographic and other analytical techniques have also been discussed.
\end{abstract}

Keywords. MALDI; mass spectroscopy; gel permeation chromatography (GPC); ion fragmentation; copolymer analysis.

\section{Introduction}

The chemical structures of polymers are characterized typically by determining the repeat units i.e. monomers, end-groups that cap the polymer chains and the molecular weight distribution. Polymer characterization with mass spectroscopy, MS, is not possible as MS requires gasphase ions for a successful analysis and polymers are composed of large, entangled chains that are not easily converted to gas phase ions. Traditional mass spectroscopic techniques developed for polymer analysis, like pyrolysis gas chromatography use thermal energy to vapourize non volatile samples like polymers. Although thermal energy also decomposed polymers into constituent parts leading to fragmentation, the full chemical structure information used to be lost during vapourization.

A new technique has been developed to measure this aspect of polymer sample. This new technique is known as matrix assisted laser desorption ionization mass spectroscopy (MALDI MS). It is perhaps the most important mass spectroscopy method used to analyse polymer systems. It is a special case of MS, using specific sample preparation methods and low fluence laser desorption to create the analyte ions. It was invented by Tanaka and his co-workers (Karas and Hillenkamp 1988; Tanaka et al 1988; Bahr et al 1992). MALDI MS has rapidly grown in applications ranging from sequencing peptides to measuring the average molecular weights of complex synthetic polymer materials.

*Author for correspondence

(jagtap_68@yahoo.com, Jagtap@udct.org)
The use of lasers for generating ions began from the early 1960s. These ions were used for analysis in MS (Vastola et al 1970). Researchers have used a wide variety of lasers of different wavelengths and pulse widths and tried to combine them with various spectrometers. The first systematic attempt to generate ions from organic molecules began from the early 1970s (Posthumus et al 1978; Kupka et al 1980). Numerous systematic observations led to two general principles. First, efficient and controllable energy transfer to the matrix, lasers emitting in the far ultraviolet which can couple to electronic states, or far-infrared, give the best results. Second, to avoid thermal decomposition of the thermally labile molecules, the energy must be transferred in a very short time. Usually, lasers with pulse widths in the range of $1-100 \mathrm{~ns}$ are used. These lasers can be easily focused on small spot sizes that are comparatively smaller than the other dimensions of the ion source and hence are essentially generated at a point source in space and time. This makes these lasers easily compatible with time of flight (TOF) mass spectrometers.

Early experiments on laser desorption of organic ions revealed that there was an upper limit on the size of the molecules that can be desorbed as intact ions. This limit was dependent on the molecular structure and was $1000 \mathrm{Da}$ for biopolymers and $9000 \mathrm{Da}$ for synthetic polymers and laser parameters. These limitations were due to the resonant excitation of the analyte molecules, necessary for the controllable non-explosive energy transfer and also put energy into the photo dissociation channels. In nonresonant desorption experiments, the necessary irradiations are very close to the onset of plasma generation, 
which destroyed the large organic molecules. An important breakthrough towards the higher mass organic molecules was achieved by Karas et al (1987) who observed that the use of matrix could avoid this problem. Thus, the concept of MALDI MS came into existence.

\section{Principle of MALDI MS}

MALDI is a soft ionization technique in which the energy from the laser is spent in volatilizing the matrix rather than degrading the polymer. Figure 1 elucidates the principle of this technique.

This technique is based upon an ultraviolet absorbing matrix. The matrix and the polymer are mixed at a molecular level in an appropriate solvent. The solvent helps prevent aggregation of the polymer. The sample-matrix mixture is placed on the sample probe tip, under vacuum conditions; the solvent is removed, leaving co-crystallized polymer molecules homogenously dispersed within matrix molecules. When the pulsed laser beam is tuned to the appropriate frequency, the energy is transferred to the matrix which is partially vapourized, carrying intact polymer into the vapour phase and charging the polymer chains.
In the linear time of flight (TOF) analyser, the distribution of molecules emanating from a sample is imparted to identical translational kinetic energies after being subjected to the same electrical potential energy difference. The ions travel the same distance down an evacuated field free drift tube. The smaller ions arrive at the detector in a shorter time than the more massive ions. Separated ion fractions at the end of the drift tube are detected by an appropriate recorder that produces a signal upon impact of each ion group. The digitized data generated from successive laser shots are added up yielding a TOF mass spectrum. The TOF mass spectrum is a recording of the signal as a function of time. The time of flight (TOF) for a molecule of mass, $m$, and charge, $z$, to travel this distance is proportional to $(\mathrm{m} / \mathrm{z})^{1 / 2}$. This relationship can be used to calculate mass of the ions. Through calculation of mass of the ions, conversion of TOF mass spectrum to a conventional mass spectrum of mass to charge axis can be achieved as shown in figure 2 .

\section{Advantages of MALDI MS}

(I) One of the key advantages of MALDI MS is that the absolute molecular weights of oligomers can be deter-

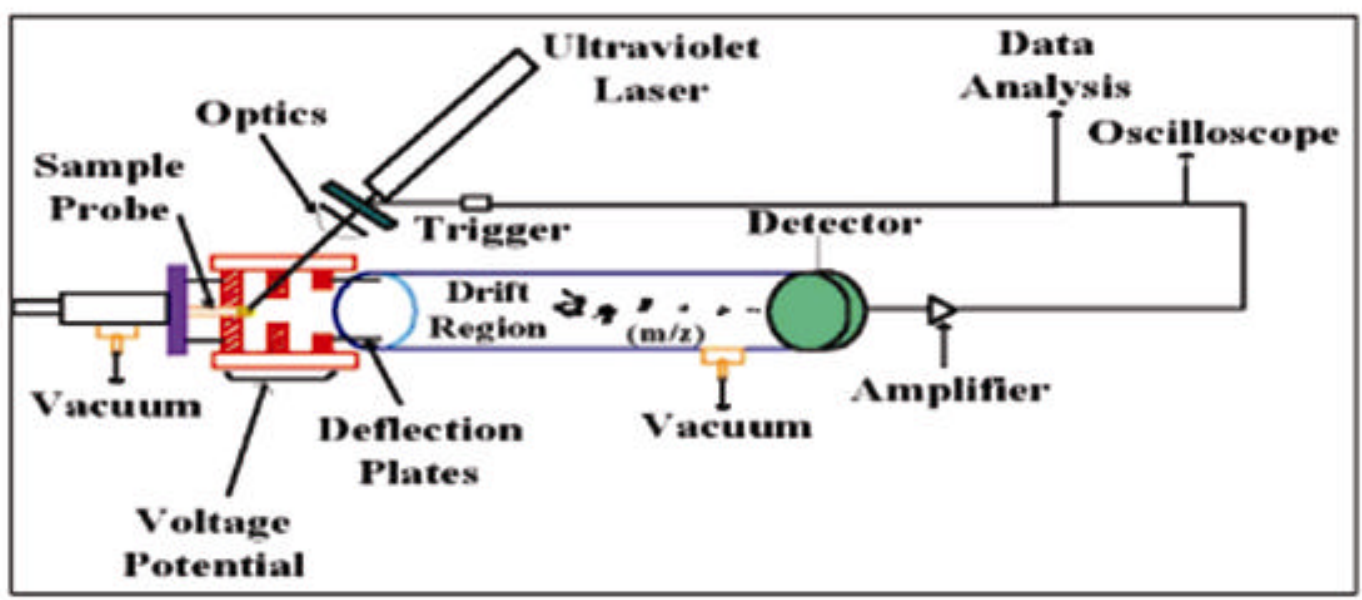

Figure 1. Principle of MALDI.

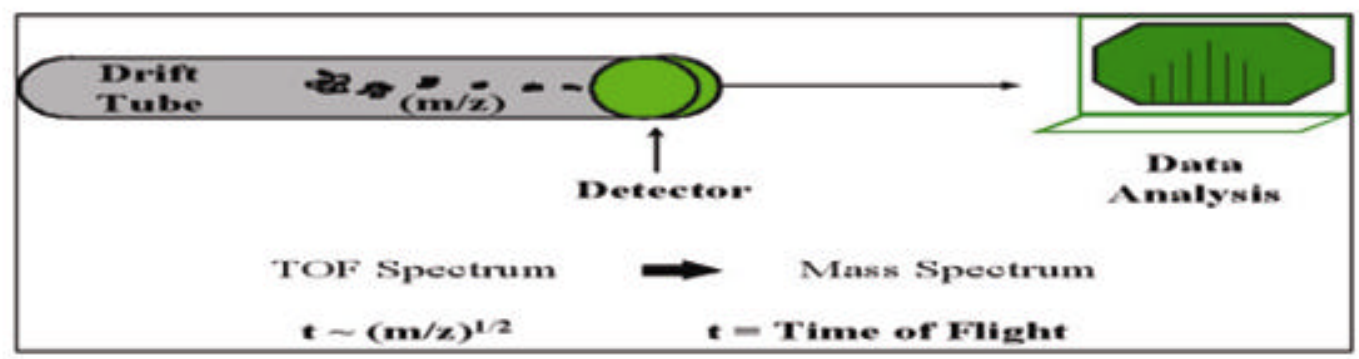

Figure 2. Conversion of TOF spectrum to mass spectrum. 
mined as opposed to obtaining relative molecular weights by chromatographic techniques. MALDI MS does not require polymer standards to assign molecular weights to oligomers, and the technique permits accurate determination of molecular weights from narrowly distributed polymers with polydispersity $<1.2$ (Kuang and Odom 1998).

(II) The speed and information obtained by MALDI MS are significantly more than that obtained with other conventional molecular weight determination techniques.

(III) MALDI MS can determine molecular weight independent of the polymer structure. For example, rigid rod polymers such as tetrahydropyrene are very difficult to analyse by conventional analysis technique such as gel permeation chromatography because these polymers have stick-like geometry. Most polymers have flexible backbones that form random coil conformations in solution. These polymers are readily analysed by GPC methods, which are based on relating the hydrodynamic volume of the coiled polymer chains with molecular weight. However, the hydrodynamic volume of the rigid rod polymers does not correlate with molecular weight. Hence molecular weights determined by gel permeation chromatography often deviate from the true values for such polymers.

(IV) MALDI MS can be used to analyse materials that are difficult to characterize by GPC methods, because of either solvent or column incompatibilities. Moreover, GPC does not perform well in low-molecular-weight regions (masses $<20 \mathrm{kDa}$ ), whereas MALDI MS works very effectively in this mass range. Polymer characteristics that can readily be determined by MALDI MS include molecular weight averages (including the number $\left[M_{\mathrm{n}}\right]$ and weight $\left[M_{\mathrm{w}}\right]$ averaged molecular weights), polydispersity, mass of repeat units, and end-group mass structure.

(V) Molecular weight and polydispersity data can be used to verify synthetic pathways, study degradation mechanisms, look for additives and impurities, and compare product formulations. End-group and chemical structure data are critical to understanding structure-property relationships of polymer formulations. MALDI MS provides a direct method of determining end-group mass and composition as well as the mass and composition of oligomer repeat units.

(VI) MALDI can be used to determine the molecular weight of polymers having mass $>200000$ Da by ionization and evaporation without any degradation of the polymer.

\section{Limitations of MALDI MS}

(I) MALDI has problems in measuring the molecular weight of polymers with polydispersity above 1.6. This problem is a combination of several factors such as sample preparation, laser fluence, instrument dynamic range, and fragmentation and detection efficiency (Hanston 2001).
(II) Some polymers such as teflon, polyimide and polyurethane still cannot be characterized by MALDI MS (Analytical Chemistry News and Features 1998).

(III) It is difficult to analyse complex mixtures of surfactants. Quantization of mixtures is quite difficult (Hanston 2004).

(IV) MALDI MS is also challenged by chemistry that has too little functionality to stabilize a cation in the gas phase. For example, saturated hydrocarbons like polyethylene and polypropylene are difficult to analyse by MALDI (Analytical Chemistry News and Features 1998; Hanston 2004).

\section{Sample preparation}

The sample preparation for polymer MALDI must accomplish five different roles (Analytical Chemistry News and Features 1998; Hanston 2004), one for the solvent and four for the matrix. The roles are as follows

- The solvent must effectively separate the polymer molecules. We need to minimize the interactions between the polymer molecules and generate individual molecules for the MALDI experiment to analyse.

- Isolate the oligomers: The matrix must maintain the separation of the oligomers obtained by dissolving the polymer in a good solvent.

- Absorb energy: The matrix must absorb energy delivered to the sample, usually wavelength of $337 \mathrm{~nm}$ of the laser.

- Desorb the analyte: The matrix must convert the energy delivered by the laser to eject the molecules in the gas phase.

- Ionize the analyte: The matrix must provide an ionization path for the analyte molecules.

\subsection{Different methods of sample preparation}

5.1a Dried droplet method: In this method, a dilute solution of analyte is prepared in a good solvent (Dale et al 1997; Hanston 2001). This analyte solution is then mixed with a more concentrated matrix solution in the same solvent. The solvent must be selected carefully to be a good one for both the analyte and the matrix. A relatively volatile solvent can be used. The mix ratio of the two solutions should result in a matrix to analyte ratio between 100 and 10000.

For low molecular weight polymers we typically use $5 \mathrm{mg} / \mathrm{mL}$ polymer solutions and mix them $1: 7$ with $0 \cdot 25 \mathrm{M}$ matrix solutions. About $1 \mu \mathrm{L}$ of the resulting solution is then deposited on the target substrate and dried and the solid mixture is then placed into the mass spectrometer.

5.1b Layer method: The matrix solution is applied to the target surface and allowed to dry. The sample solution is then applied to the dry matrix crystals (Hanston 2001). 
Some samples require more control over the evaporation process to achieve successful sample preparation. The evaporation can be controlled by electro spraying or pneumatically spraying the sample on the substrate. The spraying techniques control the evaporation of the solvent to obtain improved mixing of the analyte and the matrix. This is especially important for analytes that have relatively poor solubility in the matrix and for experiments where quantization is important.

5.1c Fast-evaporation method: The fast-evaporation method was introduced with the main goal of improving the resolution and mass accuracy of MALDI MS measurements. Matrix and sample are handled separately. This method is similar to the layer method with a difference that the process delivers stable and long lived matrix films that can be used to precoat MALDI targets.

5.1d Electrospray: A small amount of matrix-analyte mixture is electro sprayed from a $\mathrm{HV}$-biased $(3-5 \mathrm{kV})$ stainless steel or glass capillary onto a grounded metal sample plate, mounted $0 \cdot 5-3 \mathrm{~cm}$ away from the tip of the capillary. Electrospray sample deposition creates a homogenous layer of equally sized microcrystal and the polymer molecules are evenly distributed in the matrix. The method has been proposed to achieve fast evaporation and to effectively minimize sample segregation effects.

5.1e Matrix-precoated layers: This sample preparation method is reduced to the straightforward addition of a single drop of undiluted sample to a precoated target spot. The advantages are faster preparation and more sensitive results than the previous methods. It also offers the opportunity to directly interface the MALDI MS sample preparation to the output of LC and CE columns. Most efforts have been focused on the development of thinlayer matrix-precoated membranes e.g. nylon, PVDF, nitrocellulose, anion- and cation modified cellulose, regenerated cellulose or regenerated cellulose dialysis membrane.

5.1f Chemical liquid: The preparation of such samples is rather simple. An appropriate molar ratio of the analyte is dissolved in the liquid matrix, often by employing a solvent that is evaporated before introduction of the sample into the vacuum system of the mass spectrometer.

5.1g Particle-doped (two-phase) liquid: In the case of two-phase matrices, a suspension of particles (fine metal or graphite with a diameter of $\leq 1 \mu \mathrm{m}$ ) in a solvent is mixed with analyte and binder. The solvent is evaporated and the remaining paste on the sample holder is introduced in the ion source. Care should be taken about possible contamination by small particulates that are sputtered from such samples. The fine particles absorb most of the energy from the laser beam and promote the desorption. The liquid molecules provide the charge for ionization. Several combinations of particulates and liquids have been used to analyse polymers, and dyes up to a molecular weight of over 10,000.

\section{Choice of the matrix}

The key to MALDI MS experiments is the matrix. In each experiment, we have four important roles to accomplish to produce ions: intimacy, absorption, desorption and ionization (Hanston 2004).

Intimate contact between the matrix and the analytes is required to produce ions. The matrix should readily mix with the analyte molecules. They should have the same hydrophobicity or hydrophilicity to the analyte. Some common matrices used for MALDI experiments are given here.

In recent years a few matrices have proven most popular. While some researchers prefer to employ their own mixtures, most have now settled on a number of standard choices.

As a general rule, one should try to match the solubility of the matrix and the sample, since the MALDI effect depends upon the close proximity of the two generated by co-crystallization for charge transfer to take place. Table 1 shows the ranks of common matrices and polymers by their hydrophobicity (Hanston 2001).

Table 1. Ranking of polymers and their matrices by their hydrophobicity.

\begin{tabular}{lll}
\hline Matrices & & \multicolumn{1}{c}{ Polymers } \\
\hline & Hydrophilic & \\
Thiourea & & Polyethylene glycol \\
Dihydroxybenzoic acid & Polypropylene oxide \\
Cyano-hydroxy-cinnamic acid & & Polyvinyl acetate \\
Ferulic acid & & Poly(tetramethylene glycol) \\
Indole acrylic acid & Polymethylmethacrylate \\
Dithranol & Polystyrene \\
Retinoic acid & & Polybutadiene \\
Diphenyl butadiene & Polydimethylsiloxane \\
& Hydrophobic & \\
\hline
\end{tabular}


The analyte molecules need to be in close proximity to the matrix molecules to be effectively desorbed. The only source of energy to induce desorption is the laser light. The matrix must absorb at the wavelength of laser. Most commercial MALDI instruments have ultraviolet nitrogen lasers with emission at $337 \mathrm{~nm}$. To volatilize the analyte, the matrix must assist the desorption of the analyte from the substrate.

Upon irradiation by the laser pulse, the matrix molecules absorb most of the laser's energy. The high matrix to analyte ratio ensures that most of the energy is absorbed by the matrix and minimizes direct irradiation of the analyte. The energy absorbed by the matrix molecules is utilized for electronic excitation of the matrix within the solid sample mixture, creating an instantaneous phase transition from a solid phase to the gas phase.

The amount of material removed by each laser pulse is estimated to be approximately about $10-100 \mu \mathrm{m}$ in diameter (laser spot size) and a few hundred nanometers deep. A dense gas cloud is formed and expands supersonically (at Mach 3) into the vacuum. It is believed that analyte ionization occurs in the expanding plume as a direct result of collision between analyte neutrals, excited matrix ions, and protons and cations such as silver and sodium.

The matrix must also facilitate ionization of the analyte molecules. MALDI ions are not formed directly by the absorption of laser light. Primarily we observe cat ionized species. Since most matrices are acidic, protonation is a common mechanism for analytes with basic functional groups. Another mechanism is metal cat ionization. Analytes with oxygen functionality are readily cat ionized with alkali metal salts like $\mathrm{LiCl}, \mathrm{NaCl}, \mathrm{KCl}$ and analytes with unsaturated hydrocarbon functionality are readily cat ionized with transition metals like silver and copper. Addition of metal cations is a part of the sample preparation method.

\section{Choice of the solvent}

The choice of the solvent also plays a very important role in MALDI analysis. Commonly used solvents are acetone, hexane, methylene chloride, tetrahydrofuran and chloroform.

A polymer sample may be soluble in all the solvents mentioned above. The solvent which produces narrow polydispersity mixture with the analyte polymer sample should be chosen. These narrow polydispersity sample mixtures are ideal for analysis by MALDI.

\section{Applications of MALDI MS}

\subsection{Synthetic polymer analysis}

The solubility of matrix and polymers in the same solvent is critical for polymer analysis. Some non-aqueous poly- mers can simply be dissolved in acetone or methanol in order to get MALDI signals.

Synthetic polymers which can be analysed by MALDI are classified into the following four groups (Kuang and Odom 1998): (I) Water soluble polymers: poly (acrylic acid) and poly (ethylene glycol); (II) polar organic soluble polymers: acrylics and poly (methyl mathacrylate); (III) non-polar organic soluble: polystyrene, polyethylene and (IV) low solubility polymers: cured polyimide.

Investigation of synthetic polymers utilizing MALDI techniques, first investigated by Karas et al (1987), included studies of poly (propylene glycol) and poly (ethylene glycol).

With the developments in MALDI methods, the technique is used to characterize the polymer reactions. MALDI data can be used to characterize both the chemical structures of the products and the rate coefficients of chemical reactions.

MALDI combined with gel permeation chromatography (GPC) were used to characterize the cyclic aryl ether ketone oligomer (Wang et al 1996). The polymerization was accomplished by reacting bisphenol A with 1,2-bis (fluorobenzoyl)-benzene under pseudo high dilution conditions. The mass spectrum revealed a series of low molecular weight oligomers and the assigned structure. Two different ions are observed for each oligomer. The extra peak was assigned as $(\mathrm{M}-\mathrm{O})^{+}$, the result of an intermolecular cyclization that forms an isobenzofuran.

MALDI and GPC were used to measure the free radical propagation rate coefficients for pulsed-laser polymerizations of methyl methacrylate and styrene (Schweer et al 1996).

\subsection{Molecular weight determination}

Figure 3 shows the resultant spectra for one of the first successful analysis (Bahr et al 1992). The spectra is for low molecular mass $(5300 \mathrm{~g} / \mathrm{mol})$. Due to the low molecular mass of the polymer, the molar mass distribution is easily derived as $58 \mathrm{~g} / \mathrm{mol}$ from the peak to peak mass increments. The values of $M_{\mathrm{n}}, M_{\mathrm{w}}$ and $M_{\mathrm{p}}$ agreed well with the values specified by the manufacturer as PPG5300 .

In another study, the MALDI technique was used to investigate a poly (ethylene glycol) sample having a high molar mass of 23000. At $\mathrm{m} / \mathrm{z} 23000$, the mass resolution is not enough for us to resolve adjacent molecular ions with a resolution of 44 mass units. There is a limit to the peak to peak resolving capabilities of MALDI which has been observed by Bahr et al (1992) to be around $20000 \mathrm{~g} / \mathrm{mol}$. This can be overcome by improving the instrumentation used for MALDI.

Polystyrene was another example studied by Karas et al (1987). The sample had a mass of $20000 \mathrm{~g} / \mathrm{mol}$. This is illustrated in figure 4. 
In the low mass region peaks for the matrix ions appear. The spectra have a continuous distribution due to the convolution of the matrix ions. The molar mass for individual oligomers is seen to be $104 \mathrm{~g} / \mathrm{mol}$ from the peak to peak mass increments.

This sample utilized 2-nitrophenyl octyl ether, a viscous liquid as the matrix material because using 2,5-dihydroxybenzene matrix resulted in separation of the matrix and the polymer.

Certain condensation polymers have been investigated using MALDI MS. Contrary to most polymerization products, condensation polymers tend to possess low molar masses. However, it is the complex polymer structure of most condensation polymers that renders them interesting for MALDI MS studies. MALDI MS studies of condensation polymers have included phenolic resins (Pasch et al 1996), epoxy resins (DesMarteau and Bolinger 1996; Kosman et al 1996) and polycarbonates (Mandel and Kornfeld 1996; Pasch et al 1996), all of which are important technical products. Pasch et al (1996) studied numerous samples of the above polymers, including two commercially available oligocarbonates. MALDI MS samples of the two oligocarbonates, containing the matrix material of dithranol and $\mathrm{LiCl}$ and the oligocarbonate were mixed on a molecular level in a THF solvent solution and homogeneously vacuum co-crystallized. $\mathrm{LiCl}$ was added as part of the matrix to increase the formation of cationized species. Spectra A and B in figures 5 and 7 (Pasch et al 1996) are the resultant distributions derived from MALDI-TOF MS analysis of the oligocarbonates.

Spectra A have a straightforward molar mass distribution with peak-to-peak mass increments of $254 \mathrm{~g} / \mathrm{mol}$, equalling exactly the mass of the repeating unit of bisphenol A-based oligocarbonates.

PEG is a very biocompatible polymer (Harris and Zalipsky 1972). In aqueous solutions, PEG is a very mobile, conformationally flexible, random coil. Every ethylene oxide repeating unit of PEG is known to bind 2-3 water molecules. These properties, combined with its very low toxicity, lack of immunogenicity, and ease of excretion from living organisms, make PEG unique and popular as a biomaterial.

When grafted onto a surface, PEG causes exclusion, or steric stabilization, which is repulsion of proteins and

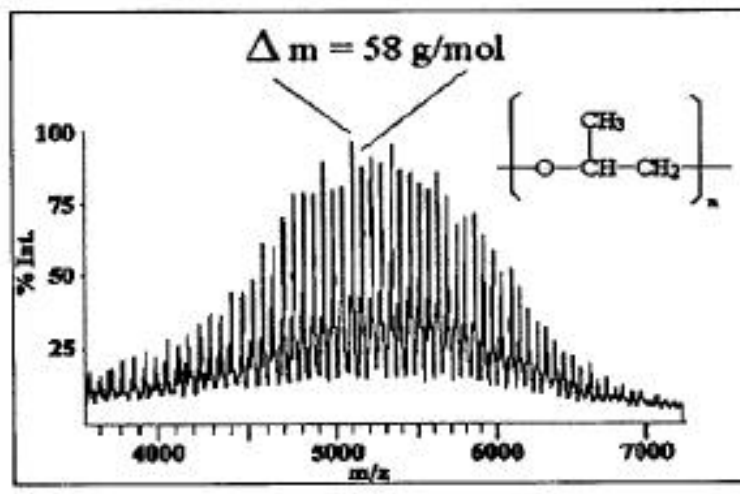

Figure 3. MALDI mass spectra.

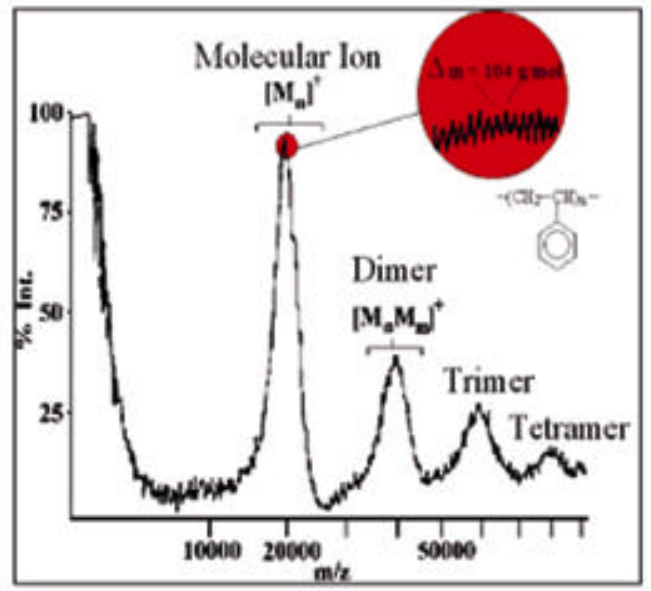

Figure 4. MALDI mass spectra.
Polymer: Poly (propylene Glycol)

Molecular weight: 5300(by GPC)

Matrix: 2, 5-Dihydroxybenzoic acid

Solvent: Water/ethanol, 1:1(v/v)

$\mathrm{M}_{\mathrm{n}}=515$

$\mathrm{M}_{\mathrm{w}}=5227$

$\mathrm{PDI}=1.01$

$\mathrm{M}_{\mathrm{p}}=5280$
Polymer: polystyrene

Molecular weight: 20000 (by GPC)

Matrix: 2-nitrophenyl octyl ether

$\mathrm{M}_{\mathrm{a}}=19337$

$\mathrm{M}_{\mathrm{w}}=19509$

PDI $=1.01$

$M_{p}=19740$ 
particulates from the surface. This property is considered to be very valuable for any material that comes in contact with blood plasma. The steric stabilization property is thought to be directly related to the high chain mobility and conformational flexibility of the polymer in aqueous solutions. PEG is widely used for preparing bioconjugates with various biologically relevant molecules, including proteins, peptides, lipids, oligonucleotides, and low-molecular-weight drugs. These conjugates are currently under development for drug delivery and medical device applications.

Because of the low dispersity of commercially available PEG and the ability of the polyether units of PEG to

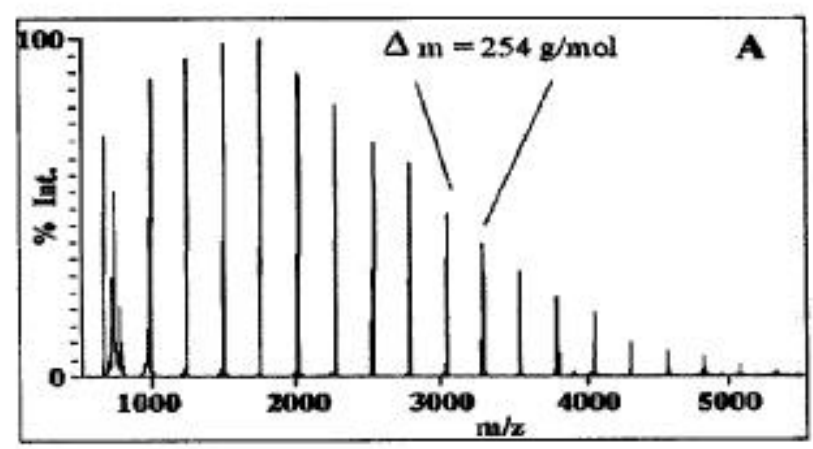

Figure 5. MALDI mass spectrum of bisphenol-A based oligocarbonates. form positive oxonium ions, MALDI MS is very valuable for characterizing reactive PEG derivatives and their conjugates. Typically, MALDI MS spectra of PEG derivatives or conjugates can be recognized by a characteristic distribution of spectral lines equally spaced at 44 mass units apart, corresponding to the molecular weight of one ethylene oxide repeat unit. Both $M_{\mathrm{w}}$ and $M_{\mathrm{n}}$ can be accurately deduced from the spectrum.

\subsection{End-group analysis}

While the bulk of a typical polymer is composed of the repeat units, the chemical structure of the end groups can be extremely important to the performance properties of any polymer material. Determining the end-groups can be helpful in determining the polymer structure.

The spectra A can also be used to investigate the endgroups of polymers. The end-group can be derived through multiple subtraction of the repeat unit from any one of the mass peaks. The end-groups of spectrum $\mathrm{A}$ in figure 5 has been derived as $228 \mathrm{~g} / \mathrm{mol}$ which corresponds to oligomers with hydroxyl end groups. The corresponding structure is shown in figure 6.

\subsection{Detection of impurities}

Spectra B in figure 7 include the same molar mass distribution with peak-to-peak mass increments of $254 \mathrm{~g} / \mathrm{mol}$,

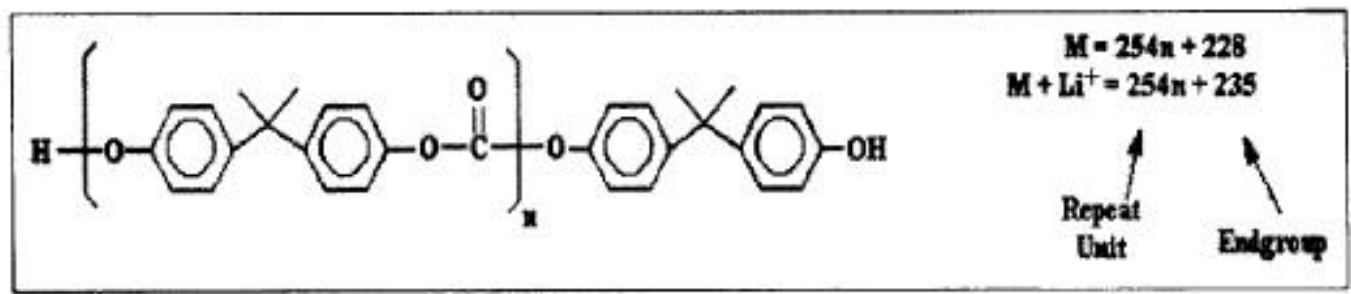

Figure 6. Possible structure of bisphenol-A based oligocarbonates with hydroxyl end-groups.

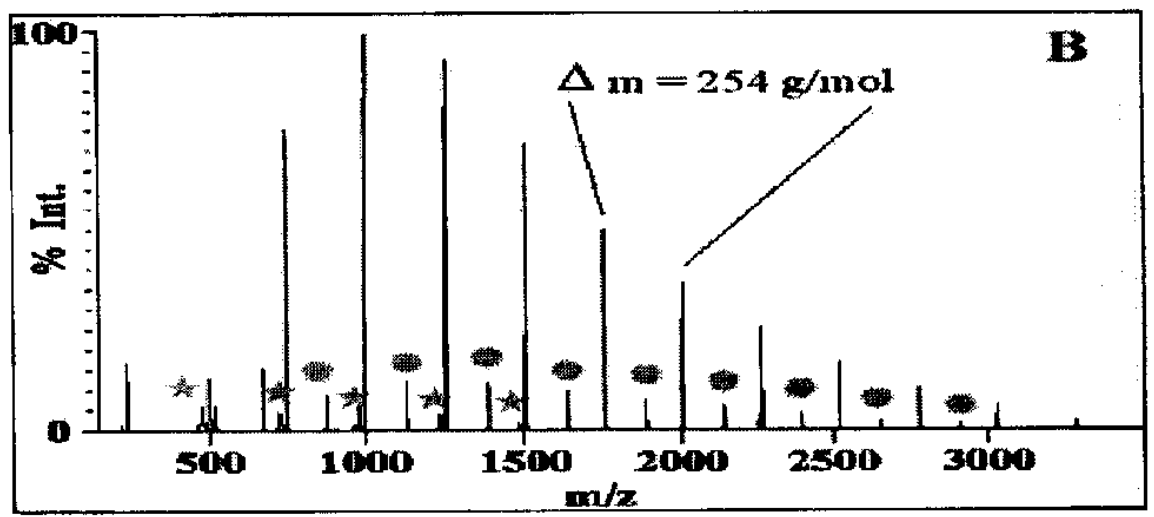

Figure 7. MALDI mass spectrum of bisphenol-A based oligocarbonates with impurities. 
equalling exactly the mass of the repeating unit of bisphenol A-based oligocarbonates. However, observance of further oligomeric series in the spectrum is significant because it is representative of inhomogeneity in the sample. The peaks in the oligomer series denoted by the above peaks are offset by $134 \mathrm{~g} / \mathrm{mol}$ from the major oligomer series. It has been suggested that these oligomers contain cresol which would be present as a purposefully added chain terminator or an impurity in the reaction mixture, as shown in figure 8 .

The peaks in the minor oligomer series denoted by the peaks with the + above them are again indicative of oligocarbonates due to the $\mathrm{m} / \mathrm{z} 254$ peak-to-peak mass increment. End group calculations allow for the speculation of the two structures as shown in figure 9.

\subsection{Copolymer analysis}

MALDI has been used mostly for the characterization of homopolymers created from a single monomer species. Materials created from two or more monomer species are copolymers. There has been comparatively little work done on copolymers due to problems in developing suitable sample preparation methods for them. The different components of the polymer must be soluble in suitable solvent to create a successful MALDI sample.

MALDI techniques are now being developed to analyse copolymers in conjunction with other analytical techniques such as gel permeation chromatography (GPC), nuclear magnetic resonance (NMR) and other mass spectral methods.

Often the MALDI data is too complex or too ambiguous due to the multiple assignments to conclusively specify the material with the mass spectral data alone.

MALDI mass spectra have been used for analysis of common copolymer surfactants composed of both ethylene and propylene oxides. The mass spectrum in figure 10 (Jackson et al 1997) shows the ion assignments as the number of EO segments and the number of PO segments for the monoisotopic ions. This is one of the easiest copolymer to analyse by MALDI because of the similarity in solubility between the EO and PO units. Series A and series $\mathrm{B}$ in figure 10 can be used to determine the chemical structure of the oligomer end groups. MALDI has also been used to analyse the chemical structure of silicon surfactants. MALDI MS was used to characterize EO or perfluoroalkyl-modified PDMS (Yoshida et al 1999). Modified PDMS surfactants are important components of many products in cosmetics industry.

MALDI was also used for comprehensive studies of diblock methylstyrene-styrene and methylstyrene-vinyl pyridine copolymers (Wilczek-Vera et al 1996).

As an example of analysis of a more complex copolymer, MALDI was used to analyse a terpolymer composed of poly (butylene succinate), poly (butylene adipate) and poly (butylene sebacate).

MALDI MS combined with size exclusion chromatography (SEC) and SEC-NMR was used to characterize a

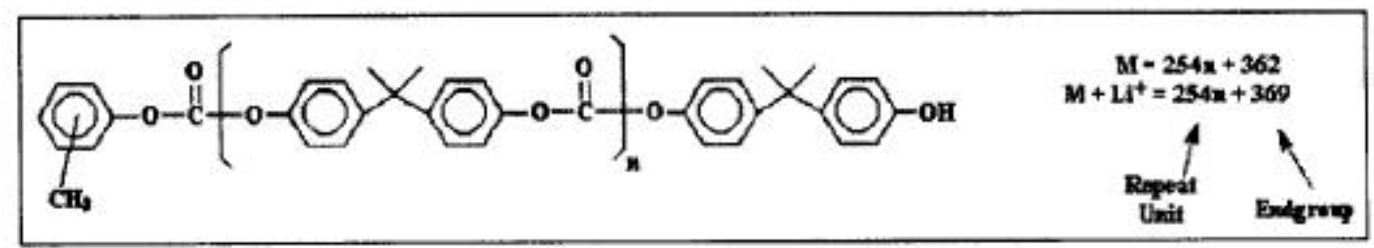

Figure 8. Detection of cresol impurities in oligocarbonates by MALDI MS.

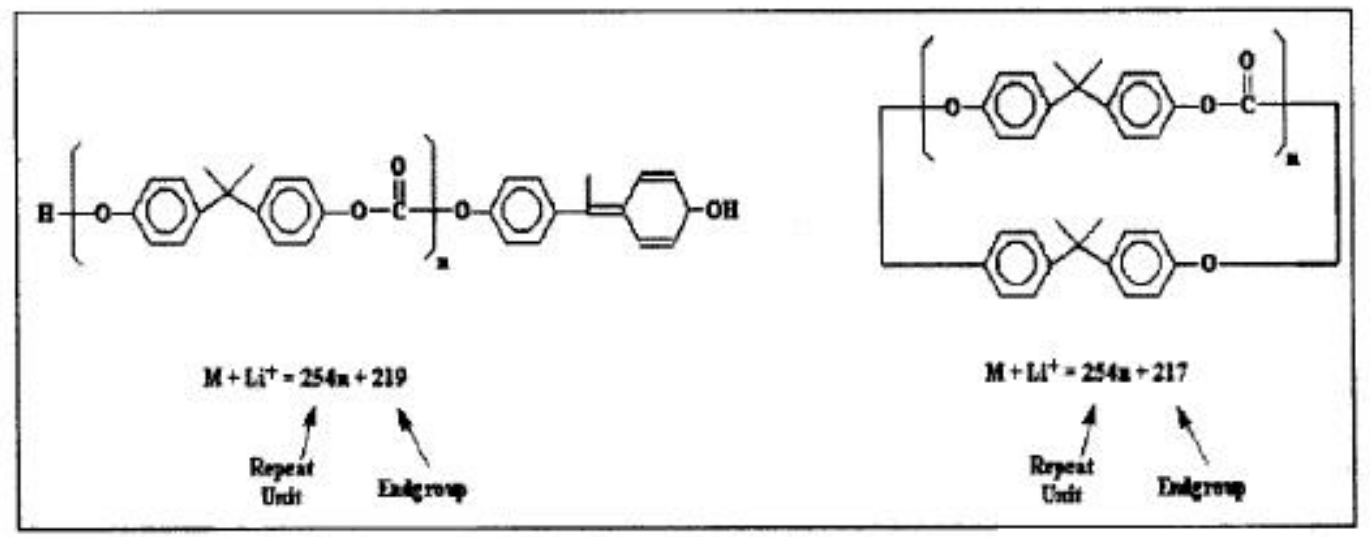

Figure 9. Two possible structures of oligocarbonates. 
series of random copolymers with units of methylmethacrylate, butylacrylate, styrene and maleic-anhydride (Montaudo 2002).

\subsection{Characterization of functional nanofillers}

Nanoparticles have attracted much attention due to their fascinating electronic, optical, magnetic and catalytic properties associated with their nano- or quantum scale dimensions (Martin and Mitchell 1998). These include metals ( $\mathrm{Au}, \mathrm{Pt}, \mathrm{Pd}, \mathrm{Cu}$ ), semiconductors $(\mathrm{CdS}, \mathrm{ZnS})$, metal and semiconductor oxides $\left(\mathrm{Fe}_{2} \mathrm{O}_{3}, \mathrm{Al}_{2} \mathrm{O}_{3}, \mathrm{TiO}_{x}, \mathrm{SiO}_{x}\right)$ and nanoparticles (Brus 1991; Wang 2000; Khomutov and Gubin 2002). Nanoparticles which do not have "spherical shape" and regular cluster sizes, include exfoliated flat silicate or clay nanoparticles 6, carbon nanoparticles, colloidal particles based $n$ pigments and polymers. Due to the high total surface area compared to microscopic and macroscopic particles, much focus has shifted to study their interfacial properties and develop new methods for their functionalization. Extensive research is being carried out to tailor the nanoparticle surfaces by the use of polymers.

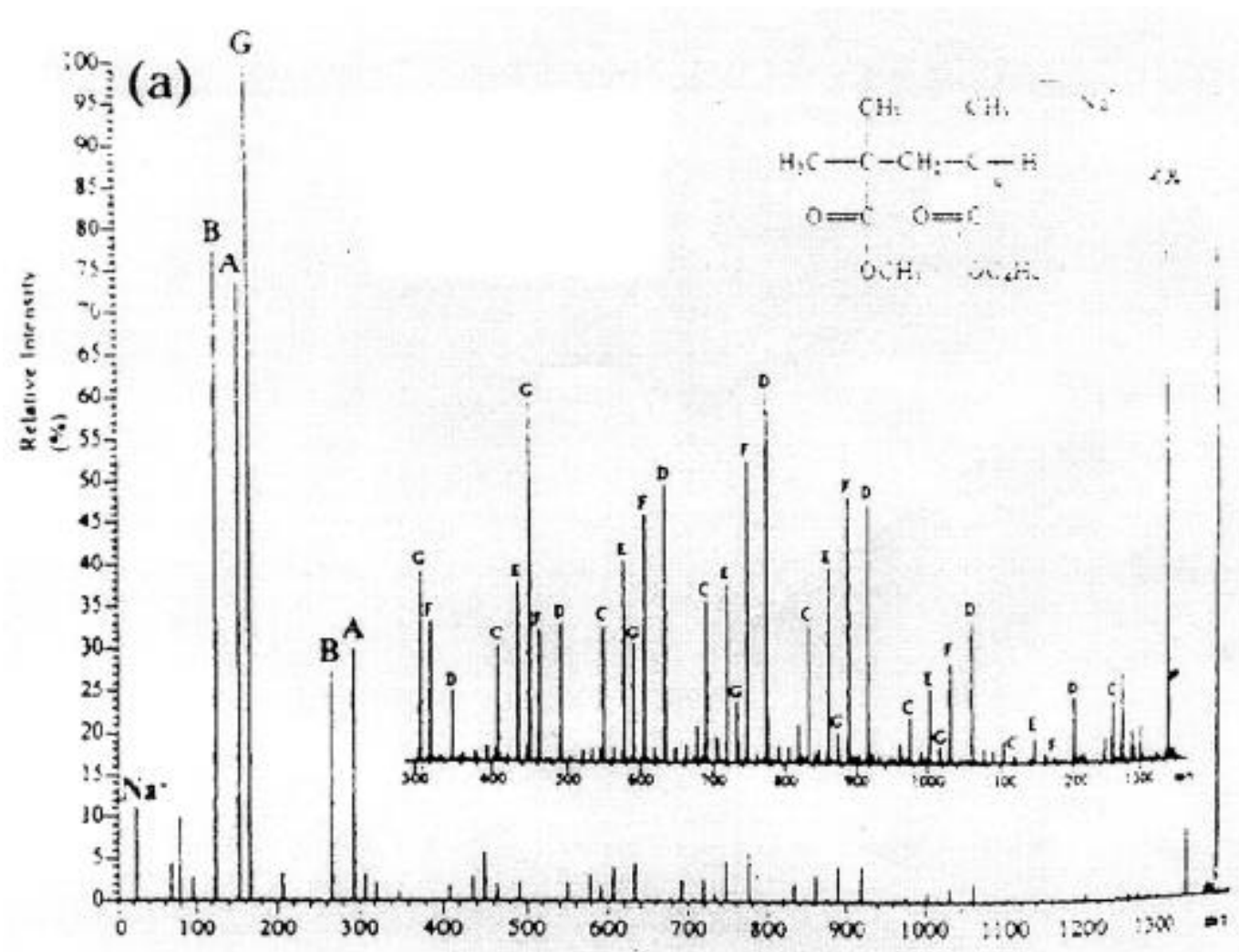

(b) B Series

A Series<smiles>COC(=O)C(C)(C)CC(C)(CC(C)(CC(C)(CC(C)(CC(C)(C)C(=O)OC)C(=O)OC)C(=O)OC)C(=O)OC)C(=O)OC</smiles>

Figure 10. MALDI mass spectrum obtained on an FTMS instrument of a common ethylene oxide/ propylene oxide copolymer. 
One of the methods to tailor nanoparticles is to tether polymers on the surface of these nanoparticles resulting in a core-shell type of structure. This method leads to the formation of "hairy nanoparticles" which has the effect of protecting the nanoparticle surface and increasing its compatibility to polymeric matrices. This is important for the preparation of polymeric nanocomposites. Surface initiated polymerization can be used for tethering the polymers on the surface. MALDI MS combined with gel permeation chromatography (GPC) can be used for studying the functionality of the end groups, chemical composition and the structure of the branched polymers on the surface of nanoparticles.

The inorganic nanofillers like silica used for preparing nanocomposites are dispersed easily in the polymer matrix if they have certain functional groups grafted on their surface. These functional groups increase the adhesion of silica particles with the polymer matrix and impart improved mechanical, optical and thermal properties.

MALDI-TOF was used to study the grafting of silanes on silica nanoparticles (Bauer et al 2003). The silanes used were methacryloxypropyltrimethoxysilanes (MEMO) and $n$-propyltrimethoxysilanes (PTMO). The corresponding MALDI-TOF spectrum is shown in figure 11 (Bauer et al 2003). Different polymers with a characteristic repeating mass unit 188 and $104 \mathrm{~g} / \mathrm{mol}$ are observed for MEMO and PTMO, respectively. The molecular weight distributions observed for the polysiloxanes confirm the presence of a polymeric shell around the nanoparticles.

\section{Ion fragmentation}

In MALDI technique the ions are intact showing very less fragmentation. The fragmentation is absent in most of the cases. The absence of fragmentation is advantageous for the determination of average molecular weight

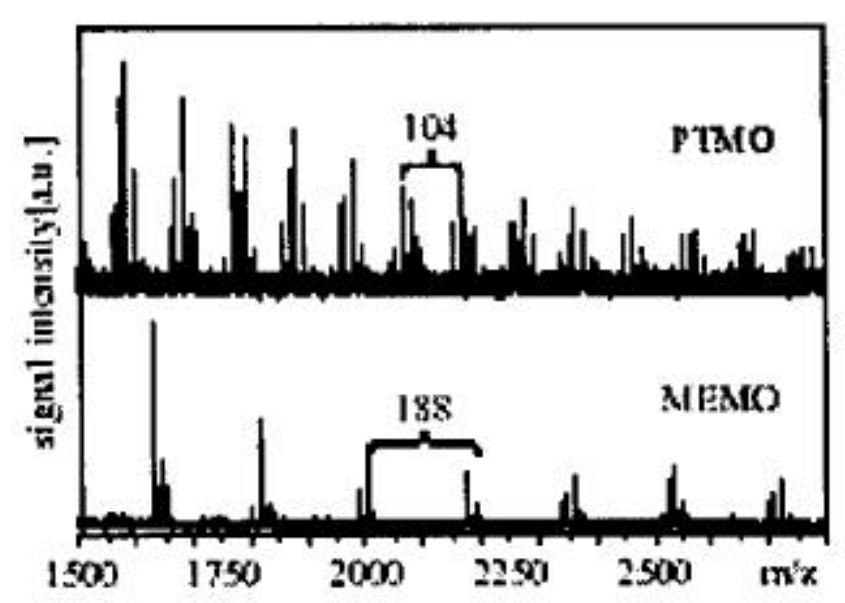

Figure 11. MALDI-TOF mass spectrum of silica nanoparticles modified PTMO and MEMO. All molecules were observed as $\mathrm{Li}^{+}$attached ions. and end-group analysis. In order to study the chemical structure, it is necessary that ion fragmentation occurs to look at the chemical connectivity in the ions.

Multiple stages of mass analysis in a single experiment have long been used to determine the chemical structure of analyte molecules. In multiple stages of mass spectroscopy experiments, the first stage of analysis is used to select a specific mass range to examine in a second stage of mass analysis. Between the two stages of mass analysis, additional energy is added to the system to fragment the ions of interest. These fragmented ions are mass analysed in the second stage.

Multiple stages of mass spectroscopy are possible with the help of collision induced dissociation (CID) and postsource decay (PSD) experiments.

In $\mathrm{CID}, \mathrm{He}, \mathrm{Ar}$ or $\mathrm{Xe}$ atoms are used for collisions with the ions. The ions dissociate because of conversion of part of the translational energy of the ion to internal energy in the ion during collision. Fragmented ions are observed which help in the determination of the polymer repeat units and end groups. Jackson and co-workers studied the MALDI-CID of poly (butyl methacrylate) at collision energy of $100 \mathrm{eV}$ in Xe (Jackson et al 1997). The CID pattern produces a rich fragmentation spectrum. The fragmentation pattern showed how the intense low mass high intensity ions labelled $\mathrm{A}$ and $\mathrm{B}$ can be used to determine the chemical structures of oligomer end groups. Pastor and Wilkins (1998) studied MALDI experiments on PEG, PS by using FTMS instruments.

The development of MALDI-PSD provided a more easy way to understand MALDI multiple stages mass spectroscopy experiments, as these experiments can be done on most commercial time of flight (TOF) reflectron experiments available today and do not require the complex hybrid magnetic sector or FTMS experiments used to do the CID experiments discussed above.

Post source decay experiments have been used to create fragmentation. In MALDI post source decay experiments the ions of interest are selected and fragmentation is initiated by increasing the number of collisions during the desorption process. The number of collisions increased by increasing the amount and intensity of laser light delivered to the sample. The increased amount of laser increases the amount of material desorbed from the sample and thus more collisions are produced between the MALDI ions. The ion fragments are not photo-induced. We can measure the fragmented ions by changing instrument voltage settings.

MALDI PSD has been applied to numerous biological molecules, but reports on synthetic polymer analysis are rare. Pryzbilla and co-workers (1999) investigated PSD of polycarbonates. Scrivens and co-workers (1997) investigated the PSD of PMMA. In both the polycarbonate and PMMA experiments, the fragment ions could be used to better understand the chemical structure of polymer end groups. 


\section{Combining MALDI with gel permeation chromatography (GPC)}

Although MALDI MS has been used extensively to provide molecular weight and structural and compositional information of synthetic polymers, one limitation of the technique is that it fails to provide correct molecular weight values for polydisperse polymers (Schriemer and $\mathrm{Li}$ 1997). Issues that affect the measurement of accurate average molecular weights of polydisperse polymers include sample preparation, laser fluence, instrument dynamic range, delayed extraction time, fragmentation, multiple charging, detector saturation, and relative detector response. The issues involved in measuring broadly polydisperse polymers have often been investigated by blending narrow standards. These studies show the problems inherent in quantifying polymer blends by MALDI.

A direct experimental procedure has been developed to overcome this limitation. The concept involves combining MALDI with chromatography.

GPC is a traditional method to measure the average molecular weight of polymers. GPC has the advantage that it can separate complex samples on the basis of molecular size and can readily analyse broad polydisperse samples. The disadvantage of GPC is calibrated to molecular weights for specific polymers and it has very low mass resolution. MALDI has the advantage of high mass resolution, high mass accuracy, and high sensitivity with the disadvantage of sample discrimination and inaccurate average molecular weights for samples with broad polydispersity.

Three common methods are used to combine MALDI and GPC.

I. Collection of individual GPC fractions followed by MALDI analysis of the collected fractions: This method is tedious because many samples have to be generated. The polymers which have been characterized by these methods are polyester copolymers, polymethylmethacrylate, polydimethylsiloxane, polystyrene, polybutylacrylate and polycarbonate. The MALDI data on the separated samples provided important information into the chemistry and molecular weights of the polymers.

II. Collection of continuous GPC fractions followed by MALDI: This method consists of continuously collecting the eluant on an appropriate target followed by MALDI. The key to this approach is to efficiently spray the column eluant on an appropriate target followed by MALDI. This method has been used to characterize the polymethylmethacrylate samples.

III. Online MALDI analysis of the GPC eluant: These experiments have been carried out on low molecular weight polyethylene glycol and polypropylene glycol standards (Fei and Murray 1996).

While GPC is the most prevalent technique combined with chromatography, it is not the only one. Thin-layer chromatography (Gusev et al 1995), capillary electrophoresis (Barry et al 1998), temperature gradient inverse chromatography ( $\mathrm{Ji}$ et al 2002) and gradient reverse phase liquid chromatography (Lee et al 1999) are also used.

Thin layer chromatography was used to examine the composition of ethoxylated surfactants in non-ionic detergents.

\section{Comparison of selected separation techniques for synthetic polymers and their characteristics for coupling with MALDI}

A comparative overview of these techniques is given in table 2 .

The mass range of MALDI mass spectroscopy is high and therefore, it is usually coupled with SEC. Off-line MALDI analysis provides absolute mass calibration points for the SEC chromatogram, based on the polymer itself instead of external polystyrene standards. Moreover,

Table 2. Comparison of selected separation techniques for synthetic polymers and their characteristics for coupling with MALDI.

\begin{tabular}{|c|c|c|c|}
\hline Family & Technique & Basis of separation & Off-line MALDI analysis \\
\hline 1. Liquid chromatography (LC) & $\begin{array}{l}\text { SEC } \\
\text { Gradient LC }\end{array}$ & $\begin{array}{l}\text { Molecular size } \\
\text { Chemical composition } \\
\text { Chemical functionality }\end{array}$ & $\begin{array}{l}\text { Very narrow molecular weight fractions } \\
\text { Fractions vary mainly I molecular weight } \\
\text { Optimum deposition conditions vary with time }\end{array}$ \\
\hline 2. Field flow fractionation (FFF) & $\begin{array}{l}\text { Hydrodynamic } \\
\text { chromatography } \\
\text { Thermal FFF }\end{array}$ & $\begin{array}{l}\text { Molecular size } \\
\text { Molecular size and } \\
\text { chemical composition }\end{array}$ & $\begin{array}{l}\text { Very narrow molecular weight fractions of } \\
\text { high molecular weight } \\
\text { Very narrow molecular weight fractions of } \\
\text { high molecular weight }\end{array}$ \\
\hline 3. Capillary electrophoresis (CE) & $\begin{array}{l}\text { CE } \\
\text { Capillary electro- } \\
\text { chromatography } \\
\text { Size exclusion electro- } \\
\text { chromatography }\end{array}$ & & $\begin{array}{l}\text { Very small amounts of sample } \\
\text { Deposition not straightforward } \\
\text { Same as for CE } \\
\text { Same as for CE }\end{array}$ \\
\hline
\end{tabular}


mass spectra of low molecular weight fractions can be used to infer structural information from the polymer, such as the monomer and the end groups (Nielen and Malucha 1999).

\section{Schematic set-up of coupling liquid chromato- graphy with MALDI mass spectroscopy}

The off-line MALDI coupling has limitations of fraction collection, evaporation and pipetting which are quite laborious (Nielen and Malucha 1999). Therefore, direct deposition methods in which SEC fractions and MALDI matrix are directly and automatically deposited are preferred. A direct deposition interface for MALDI-SEC has become commercially available.

A miniaturized SEC-MALDI system is shown in figure 12. It features a micro SEC system with a THF flow of $5 \mu \mathrm{L} / \mathrm{min}, \mu \mathrm{UV}$-absorbance detection and a robotic interface in which the MALDI matrix is co-axially added to the $\mu$ SEC effluent and directly spotted on to the MALDI target without the application of heat and sheath gas (Nielen 1998; Nielen and Ab Buijtenhuis 2001). The injection valve has an internal volume of 500 nanoliters and $\mu$ SEC column is a $30 \mathrm{~cm} \times 800 \mu \mathrm{m}$ internal diameter.

On-line coupling of MALDI with liquid chromatography (Zhan et al 1999) has been studied by various research groups. In most cases peptides are the target analytes. However, this method has also been used for analysis of synthetic polymers like polyethylene glycols. In this method the effluent from the HPLC column is mixed with the solution of the matrix in a T-piece, the third leg of which is connected to the MALDI ionization chamber by a capillary tube, at the end of which a stainless steel frit is glued. The LC effluent crystallizes together with the matrix on the MS side of the frit. A laser beam is used to produce the required effect by MALDI on the crystallized effluent. The idea is to continuously regenerate the interface through combined actions of solvent flushing and laser ablation.

\section{Recent developments in MALDI MS}

Polyolefins, saturated hydrocarbon polymers such as polyethylene (PE) and polypropylene (PP) are the most widely used of all synthetic polymers. Their molecular mass and molecular mass distribution (MMD) are critical in determining performance properties. The techniques currently available are not able to perform mass spectrometry on saturated hydrocarbon polymers above molecular mass of about 1000 a.m.u. that leaves in tact polymers. Polyolefins are difficult to characterize by MALDI MS due to the ineffectiveness of conventional methods of cat ionization. The lack of polar groups, unsaturation, and aromaticity excludes cat ionization methods that are used for other common synthetic polymers. A new method is introduced in which an organic cation is covalently bonded to the polymer to produce the necessary ionization for successful MALDI MS. A strong MALDI MS signal results from polymers that give no response by other methods.

The new method involves the attachment of phosphonium or ammonium ions to one of the chain ends of polyethylene (Shen et al 2002). Fractionation products of polyethylene were used for this analysis. Since the chemical modification uses the population of molecules with terminal double bonds to represent the overall population, it was critical to verify the molecular mass distribution for the molecules with double bonds, which is an

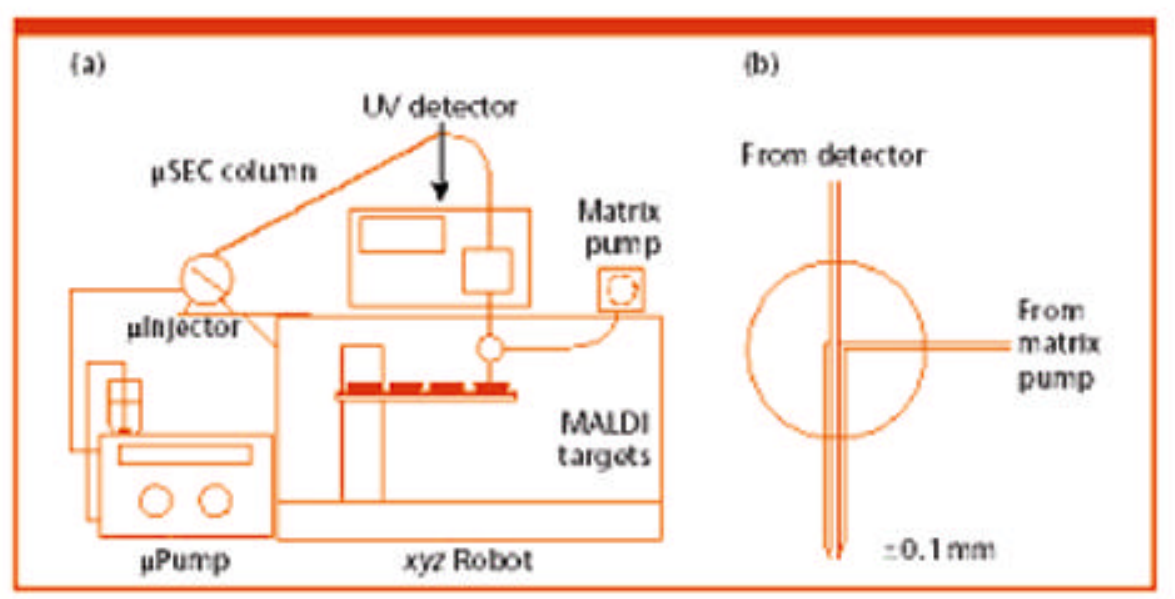

Figure 12. Set-up of $\mu$ SEC-MALDI mass spectroscopy using a robotic interface, consisting of a model 140B pump (Applied Biosystems), model C41004-0.5 injection valve (Val co cheminert), $\mu$ SEC column packed with MiniMixed-D (LC packings), a model Lambda $1000 \mu \mathrm{UV}$ absorbance detector (Bischoff), a Probot robotic interface, a Bay Bee Matrix Pump, and a Biflex MALDI MS (Bruker). 
accurate representation of the overall molecular mass distribution in each of the fractionated materials. This was verified by determining the concentration of double bonds as a function of number average molecular mass, $M_{\mathrm{n}}$.

The bromination reaction on terminal polyethylene vinyl groups produced a mixture of three products derived from different reaction mechanisms as shown in figure 13. Direct addition of $\mathrm{Br}_{2}$ across the terminal polyethylene double bond leads to the 1,2-dibromo product. Addition of hydrogen halides such as $\mathrm{H}-\mathrm{Br}$ to vinyl-terminated olefins in the absence of peroxide takes place by an electrophilic mechanism according to Markovnikov's rule and the product is 2-bromo. In the presence of peroxide, addition of $\mathrm{H}-\mathrm{Br}$ occurs via a free radical mechanism, and the orientation is anti-Markovnikov, i.e. 1-bromo.

MALDI MS were obtained for cationized covalent polyethylene. The method shows a substantial improvement in mass range and a simultaneous decrease in fragmentation compared to previously reported mass spectra of polyethylene. Moments of the mass distribution, Mn and Mw, determined from our MALDI MS data on polyethylene were lower $(60-75 \%)$ than values derived by the conventional methods of osmometry, ${ }^{1} \mathrm{H}$ NMR and light scattering. The corresponding mass spectrum of polyethylene of different grades is shown in figure 14 (Shen et al 2002).

\section{Conclusions}

Molecular weights measured by MALDI mass spectroscopy are in qualitative agreement with those obtained from other conventional methods such as GPC and low angle light scattering. Compositional information (end group and structure) that is sometimes difficult to analyse by NMR and IR spectroscopies can be derived from

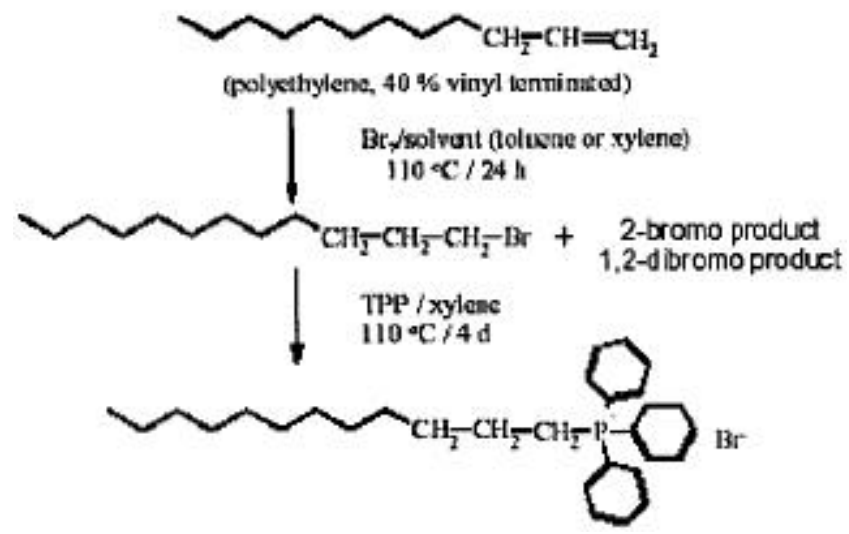

Figure 13. Bromination of polyethylene terminal double bond followed by reaction of triphenylphosphine with the 1-bromo product.
MALDI mass spectra. Ease of analysis, speed, high mass accuracy, as well as the structure independent nature of MALDI mass spectroscopy (MS) are the key advantages of MALDI for polymer analysis. The inability to analyse highly polydisperse polymers is currently a major shortcoming. This limitation can be overcome by using MALDI to calibrate GPC profiles.

By developing matrices and sample preparation methods MALDI can be used to analyse numerous polymers.
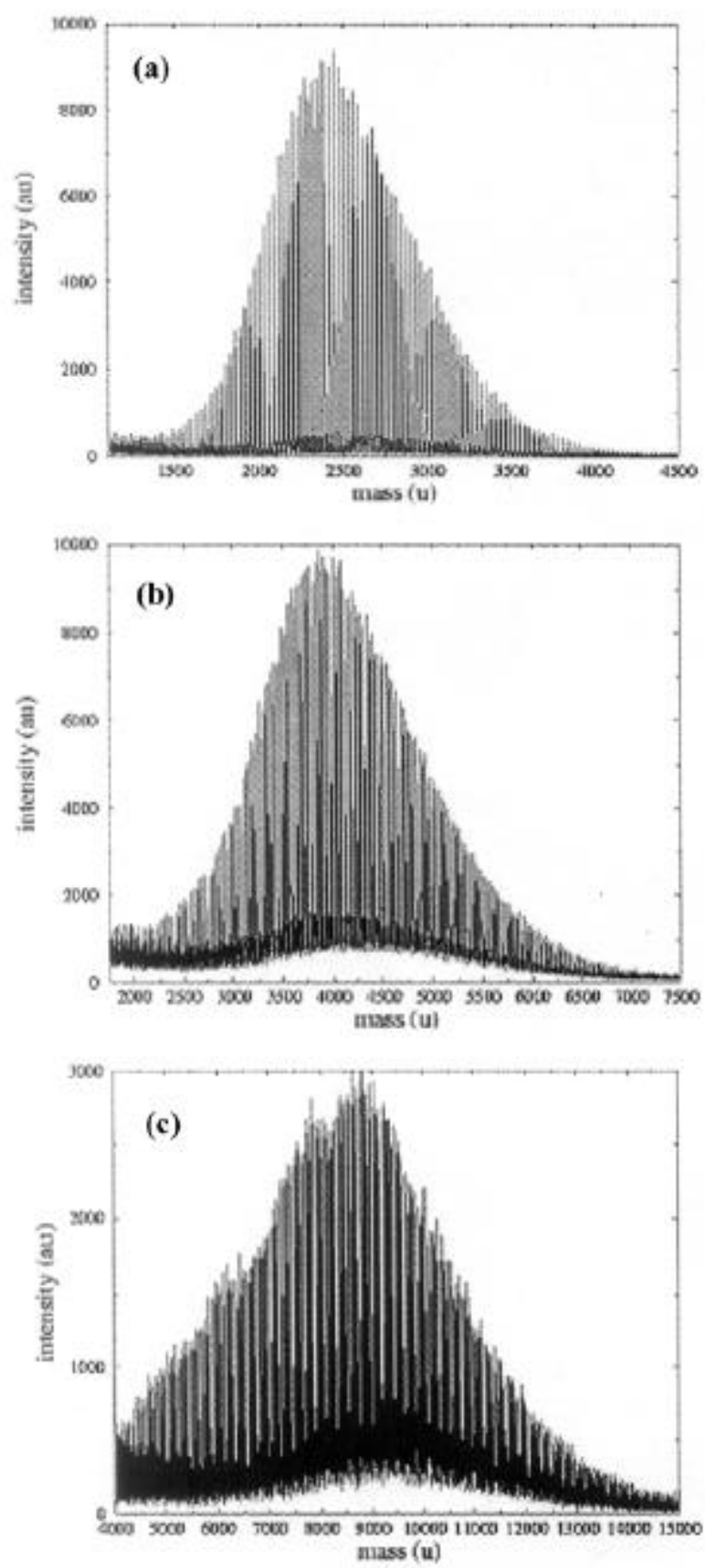

Figure 14. MALDI-TOF MS spectra of polyethylene narrow mass fraction: (a) LEA-51, (b) SRM 2885 and (c) SRM 1482. The data are shown without smoothing or background subtraction. 


\section{References}

Bahr U, Deppe A, Karas M, Hillenkamp F and Giessman U 1992 Anal. Chem. 642866

Barry J P, Radke D R, Carton W J, Anselmo R T and Evans J V 1998 J. Chromatogr. A800 13

Bauer F, Gläsel H, Decker U, Ernst H, Freyer A, Hartmann E, Sauerland V and Reiner Mehnert R 2003 Prog. Org. Coat. 47147

Brus L 1991 Appl. Phys. A (Solids and Interfaces) A53 456

Dale M J, Knochenmuss R and Zenobi R 1997 Rapid Commun. Mass Spectrom. 11136

DesMarteau D D and Bolinger M 1996 Polym. Prep. 37313

Fei X and Murray K K 1996 Anal. Chem. 683555

Gusev A I, Procter A, Rabinovich Y I and Hercules D M 1995 Anal. Chem. 671805

Hanston S D 2001 Chem. Rev. 101527

Hanston S D 2004 J. Coat. Technol. 162

Harris J M and Zalipsky S 1972 EDS, ACS symposium series 680 in Chemistry and biological applications (Washington D.C.: American Chemical Society)

Jackson A T, Yates H T, Scrivens J H, Green M R and Bateman R H 1997 J. Am. Soc. Mass Spectrum 81206

Ji H, Nonidez W K and Mays J W 2002 Macromolecules 35 1196

Karas M and Hillenkamp F 1988 Anal. Chem. 602299

Karas M, Bachmann D, Bahr U and Hillenkamp F 1987 Int. J. Mass Spectrom. Ion. Proc. $\mathbf{7 8} 53$

Khomutov G B and Gubin S P 2002 Mater. Sci. Eng. 22141

Kosman J J, Leone S M and Kornfeld R 1996 Hewlett Packard Bulletin AN96 3

Kuang J W and Odom R W 1998 Anal. Chem. A70 456

Kupka K D, Hillenkamp F and Schiller C 1980 Adv. mass spectroscopy (London) A8 935

Lee H, Nonidez Lee W, Chang T, Choi S, Lee D, Ji H, Nonidez W K and Mays J W 1999 Macromolecules 324143

Mandel F and Kornfeld R 1996 Hewlett Packard Bulletin AN96 5

Martin C and Mitchell D 1998 Anal. Chem. News and Features A322 327
Montaudo M S 2002 Polymer 431587

Nielen M W F 1998 Anal. Chem. 701563

Nielen M W F and Malucha S 1999 Rapid Commun. Mass Spectrom. 13826

Nielen M W F and Ab (F A) Buijtenhuis 2001 LC.GC Europe 1

Pasch H, Rode K, Ghahary R and Braun D 1996 Macromol. Chem. 24195

Pastor S J and Wilkins C J 1998 Int. J. Mass Spectrom. Ion Processes 17581

Posthumus M A, Kistemaker P G, Meuzelaar H L C and ten Neuver De Braw M C 1978 Anal. Chem. 50985

Pryzbilla L, Raeder H J and Muellen K 1999 Eur. J. Mass Spectrom. 5133

Schriemer D C and Li L 1996 Anal. Chem. 682721

Schriemer D C and Li L 1997 Anal. Chem. 694169

Schweer J, Sarnecki J, Mayer-Posner F, Muellen K, Raeder J and Spickrman J 1996 J. Macromolecules 294536

Scrivens J H et al 1997 Int. J. Mass Spectrom. Ion Processes 165/166 363

Shen Lin Gibson, Brunner L, Vanderhart D L, Bauer B J, Fanconi B M, Guttman C M and Wallace W E 2002 ACS Polym. Prepr. 431331

Tanaka K, Waki H, Ido Y, Akita S, Yoshida Y and Yoshida T 1988 Rapid Commun. Mass Spectrom. 2151

Vastola F J, Mumma R O and Pirone A J 1970 J. Org. Mass Spectrom. 3101

Wang Y F, Chan K P and Hay A S 1996 J. Polymer Sci. Part A; Polym. Chem. 34375

Wang Z L 2000 Characterization of nanophase materials (Verlag GmbH Weinhem, Germany: Wiley-VCH)

Wilczek-Vera G, Danis P O and Eisenberg A 1996 Macromolecules 294036

Wilczek-Vera G, Waddell K, Danis P O and Eisenberg A 1999 Rapid Commun. Mass Spectrom. 13764

Yoshida S, Yamamoto S and Takamatsu T 1998 Rapid Commun. Mass Spectrom. 8535

Zhan Q, Gusev A and Hercules D M 1999 Rapid Commun. Mass Spectrom. 12778 